\title{
Concentration Dependent Functions of FGF10 in Angiogenesis and Axon Guidance
}

\author{
Fang Liu* \\ School of Basic Medical Sciences, Medical college of Nanchang University, Nanchang, \\ Jiangxi 330006, PR China.
}

\section{A B S T R A C T}

Previous studies suggest FGF10, which is expressed in the ventral hypothalamus, plays important roles in axon guidance and in vasculature guidance of the developing hypothalamus. Here, using ex vivo analyses in chick, we show that FGF10 exerts concentration dependent functions in angiogenesis and hypothalamic axon guidance. At lower concentration of $100 \mathrm{ng} / \mathrm{ml}$, FGF10 promotes angiogenesis in chorioallantoic membrane endothelial capillaries (CAMs), but FGF10 does not alter the endothelial growth significantly at higher concentration $(500 \mathrm{ng} / \mathrm{ml}, \mathrm{P}>0.05)$. Simultaneously, FGF10 exerts a graded action, at lower concentration FGF10 attracts both magnocellular and parvocellular axons; but at higher concentration, FGF10 repels parvocellular TH+ (tyrosine hydroxylase) axons, and attracts magnocelluar vasopressin+ axons in the hypothalamus.
\end{abstract}
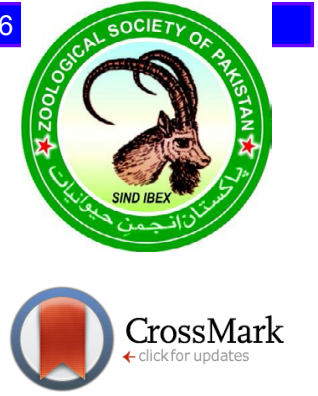

\section{INTRODUCTION}

$\mathrm{M}$ orphogens are diffusible proteins produced by restricted groups of cells. These proteins provide positional information by producing long-range concentration gradients and induce distinct responses in a concentration-dependent manner (Smith et al., 2008). FGF10 is a paracrine-signaling morphogen that has import roles during embryonic development (Itoh and Ohta, 2014). Previous studies show that FGF10 elongates the outgrowth of hypothalamic axons, and directs axons to their final targets (Liu et al., 2013). These studies reveal that low concentration of FGF10 attracts hypothalamic axons, while high concentration FGF10 repels hypothalamic axons. Nevertheless, it remains unclear the axonal guidance effects of FGF10 on specific hypothalamic axons.

Studies suggest a common mechanism for axonal and vascular growth; many axon guidance cues instruct vascular patterning, and vice versa (Eichmann and Thomas, 2013). Besides its axon guidance effects, FGF10, deriving from the ventral hypothalamus, plays an important role in endothelial outgrowth, and directs newborn vessels to the chick ventral hypothalamus (Liu et al., 2013; Liu et al., 2017). These studies focus on the vascular guidance effects of FGF10, but do not systematically interpret roles of FGF10 in angiogenesis.

\footnotetext{
Corresponding author: fangliuncueducn@126.com 0030-9923/2020/0006-2065 \$ 9.00/0

Copyright 2020 Zoological Society of Pakistan
}

Since functions of morphogens exert in a concentration dependent way, our studies for the first time compare the diverse functions of FGF10 in axon guidance and angiogenesis between high concentration and low concentration of FGF10

\section{MATERIALS AND METHODS}

\section{Explant and grafting cultures}

Explants of embryonic day (E) 4 hypothalamic neuroectoderm and chorioallantoic membrane (CAM) vessels were dispase-isolated $(1 \mathrm{mg} / \mathrm{ml}$, Roche) and cultured in collagen for 48 hours. In co-cultures, explants and beads positioned $100-200 \mu \mathrm{m}$ apart; protein FGF10 ( $\mathrm{R}$ and $\mathrm{D}$ ) was pre-soaked on Affigel beads (Pharmacia Biotech). FGF10 protein was used at high concentration $(500 \mathrm{ng} / \mathrm{ml})$ or low concentration $(100 \mathrm{ng} / \mathrm{ml})$; its antagonist SU5402 (Calbiochem) was used at $20 \mu \mathrm{M}$.

\section{In situ hybridisation}

Embryos were processed for in situ hybridization as describe previously (Vesque et al., 2000). Following development, explants (minimum 5 in each condition) were analysed as cryostat sections.

\section{Immunofluorescence analyses}

Immunohistochemical analysis of embryos $(n=6)$ and explants was performed according to standard wholemount or cryostat sectioning techniques (Vesque et al., 2000). In chick work, antibodies used were: anti-Tuj1 (anti neuron-specific class III $\beta$-tubulin; Calbiochem); anti-TH 
rabbit polyclonal (anti tyrosine hydroxylase; Chemicon); anti-Tie1 (Santa Cruz Biotechnology); anti-Vasopressin (Bachem, Torrance, CA, USA). Secondary antibodies were conjugated to Alexa 594 or Alexa 488 (Molecular Probes). Vessels were analyzed and quantitated by using Image Pro Plus 6.

\section{Statistical analysis}

Statistical analyses were carried out using SPSS19.0 for PC. Statistical significance of differences in means between groups was determined using a 2-tailed student's t-test. $\mathrm{P}$ values less than 0.05 indicates a significant difference.

\section{RESULTS}

To test roles of different FGF10 between low and high concentration in angiogenesis, we adapted the in vitro culture system, enzymatically isolated chorioallantoic membrane (CAM) endothelial vessel explants were dissected out and cultured in the 3-d collagen system. During culture in medium alone, the vessel growth was observed (Fig. 1), and these newborn vessels were Tie1+ (a marker for endothelial cells) (insets in Fig. 1). In low concentration FGF10 groups, the outgrowth detected in such control explants was extensive, and more newborn vessels were observed than vessels in control groups $(\mathrm{p}<0.01)$ (Fig. 1B; Table I). However, addition of high concentration FGF10 into the media did not appear to further promote vessel outgrowth, resulting in a similarly vessel outgrowth with control groups (Fig. 1C; Table I). Furthermore, addition of SU5402 blocked vessel outgrowth (Fig. 1D; Table I), indicating the necessity of FGFs in the growth of blood vessels.

Table I. Influence of each group on the area of newborn vessels.

\begin{tabular}{ll}
\hline Groups & Area of newborn vessels $\left(\boldsymbol{\mu m}^{\mathbf{2}}\right)$ \\
\hline Blank control & $43178.33 \pm 2786.35$ \\
FGF10 (Low) & $101338.67 \pm 9617.98^{* *}$ \\
SU5402 & $12053.33 \pm 3640.04^{* *}$ \\
FGF10 (High) & $45091.67 \pm 3690.93$ \\
\hline
\end{tabular}

Significant differences compared to control groups are shown as means \pm $\operatorname{SEM}(\mathrm{n}=6) ;{ }^{* *} \mathrm{p}<0.01 ; \operatorname{SEM}=$ standard error of the mean.

Previous studies reveal roles of FGF10 in the guidance of hypothalamic axons. To further test our hypothesis that FGF10 works on distinct hypothalamic axons in a concentration dependent way, lateral hypothalamic explants were dissected out from E4 embryos, and then
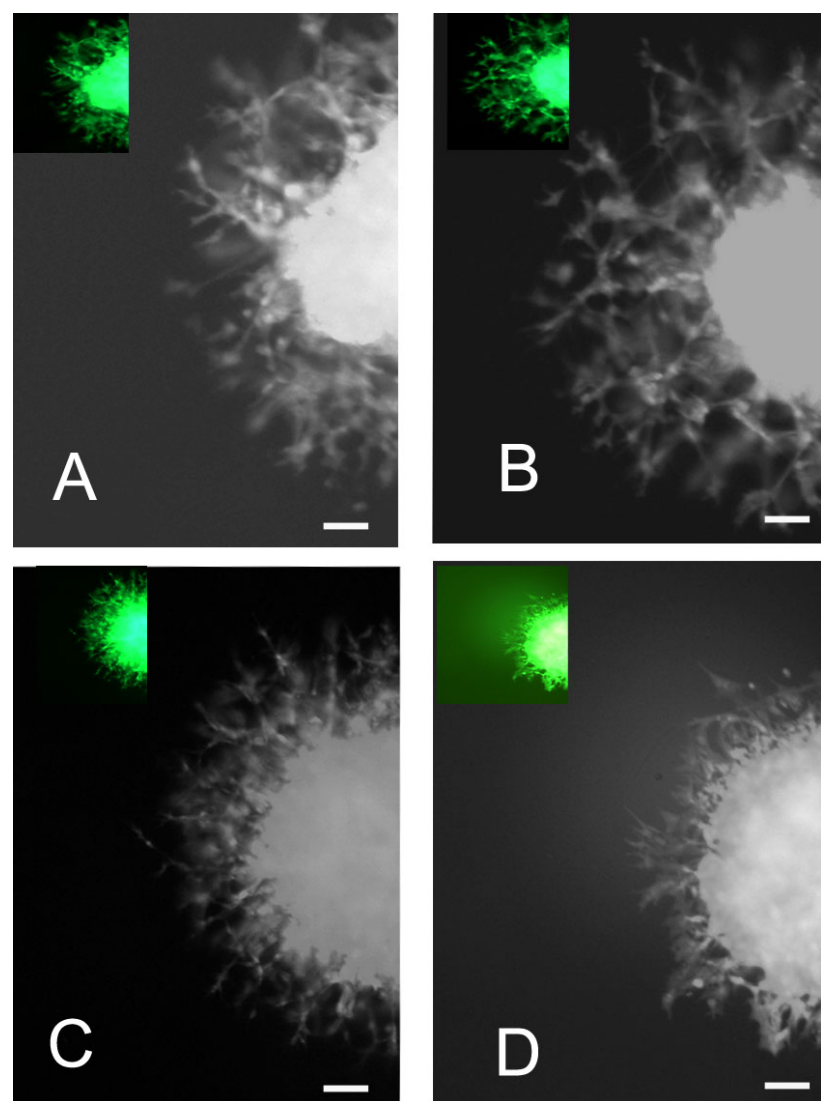

Fig. 1. Effects of FGF10 on the outgrowth of newborn vessels. (A) Control; (B) low concentration FGF10 in culture media; (C) high concentration FGF10 in culture media; (D) low concentration FGF10 plus SU5402 in culture media. Insets in A, B, C and D Show Tie-1 expression in CAM vessels. Scale bars represent $50 \mu \mathrm{m}$.

cultured with FGF10-soaked beads $(100 \mathrm{ng} / \mathrm{ml})$ in collagen gels. Explants and beads are cultured at a distance about $100-200 \mu \mathrm{m}$, and thus FGF10 beads provide concentration gradients between hypothalamic neurons and FGF10 sources. Our studies show that early-born neurons, such as vasopressin-producing neurons and $\mathrm{TH}$ (tyrosine hydroxylase) expressed hypothalamic dopaminergic (DA) neurons, are present within the E4 explants (Figs. $2 \mathrm{~A}$ and $\mathrm{B})$. The Tuj1 antibody labels neuron-specific class III $\beta$-tubulin in differentiating neural progenitor cells, and labels both early neurons and axons (Fig. 2C). After culture for two days, hypothalamic neurons extend axons in response to FGF10 beads, some axons project straight towards FGF10 sources, but other axons appear turn away from FGF10 sources (Fig. 2C). To test effects of FGF10 on distinct axonal subsets, we observe the growth of $\mathrm{TH}+$ and vasopressin+ axonal subsets. When TH axons are near the FGF10 sources, the higher concentration of FGF10 
appears to repel TH axons (Fig. 2E). However, higher concentration of FGF10 attracts vasopressin axons, and vasopressin axon goes further towards FGF10 sources (Fig. 2D).

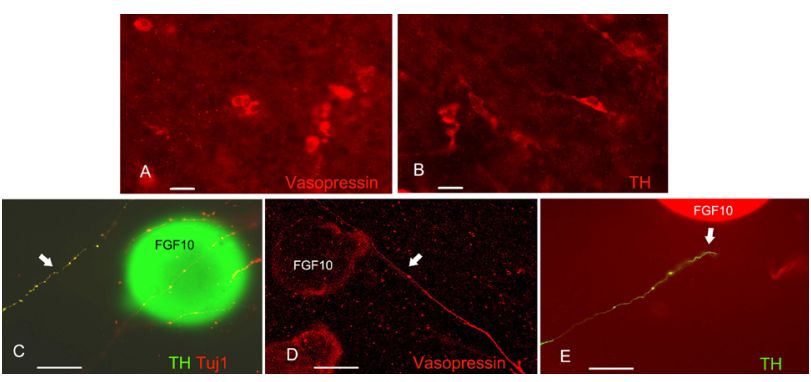

Fig. 2. Roles of FGF10 on distinct hypothalamic axons. Vasopressin (A) and TH (B) positive neurons are present within the E4 explants. After immunolabelling with Tuj1, axons extend towards FGF10 soaked beads, but one axon $(\mathrm{TH}+)$ turn (arrow), rather than project into FGF10 beads (C). Vasopressin axon goes directly towards FGF10 sources (arrow, D); while TH axon turns away from FGF10 beads (arrow, E). Scale bars represent $50 \mu \mathrm{m}$.

FGF10 belongs to the FGF7 subfamily whose receptors are FGFR1 and FGFR2 (Mason, 2007). We thus analysed the expression pattern of Fgfrl, 2 within the hypothalamus at E5. These results suggest that Fgfrl and Fgfr 2 are expressed throughout the hypothalamus, not only expressed in the ventricular zone of the lateral hypothalamus, but also expressed in the mantle zone of the hypothalamus, a zone through which hypothalamopituitary axons would project (Figs. 3A and B).
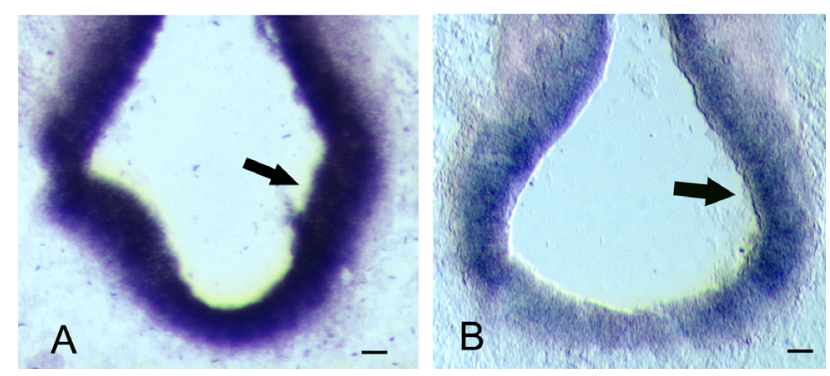

Fig. 3. Fgfrl (A) and Fgfr2 (B) expressions are detected throughout the hypothalamus (arrows), in both hypothalamic ventricular and mantle zone. Scale bars represent $50 \mu \mathrm{m}$.

\section{DISCUSSION}

FGF10 deriving from the ventral hypothalamus plays an important role in directing newborn vessels to the chick ventral hypothalamus. However, these studies do not systematically interpret roles of FGF10 in vascularisation. There are two processes during vascular network formation, vasculogenesis and angiogenesis. Vasculogenesis describes the differentiation of endothelial precursor cells from mesoderm and their colescence into tubes of central axial vessels, whereas angiogenesis is the subsequent sprouting, bridging and branching from pre-existing vessels (Ribatti, 2014). Fibroblast growth factors (FGFs) play an important role in vessel formation as a stimulatory regulator (Yang et al., 2015). Of these, the most important and frequently being studied member in angiogenesis and vasculogenesis is FGF-2. Studies indicate that FGF-2 exerts a vital role in vascular responses by increasing EC proliferation and stimulating migration, and is critical for vascular development and angiogenesis (Sahni et al., 2008). However, roles of other FGFs are not well studied as FGF2. There are 7 subfamilies of FGFs in vertebrates, which signal through FGF receptors 1-4 (FGFRs 1-4) (reviewed by Mason, 2007). FGF10 belongs to the FGF7 subfamily whose receptors are FGFR1 and FGFR2 (Brewer et al., 2016). Here, we report an investigation of angiogenic function of FGF10 in different concentrations by using in vitro assays. At low concentration, FGF10 promotes the outgrowth of newborn vessels extensively; whereas the angiogenic effects of FGF10 fade at higher concentration. Together, these studies provide preliminary data for understanding roles of FGF10 during the process of angiogenesis; and our data indicate that the angiogenic role of FGF10 is mediated through a concentration dependent way.

The hypothalamus is located in the ventral diencephalon and acts to regulate body homeostasis. One of the most important roles of the hypothalamus is to link neuroendocrine neurons in the hypothalamus to the endocrine system via the pituitary gland (hypophysis). Hypothalamic neurons that project axons to the pituitary, can be classified into two major types, parvocellular and magnocellular neurons. Parvocellular neurons of the hypothalamus synthesize and secrete hypothalamic releasing/inhibiting neurohormones, such as thyrotropinreleasing hormone (TRH), dopamine (DA), growth hormone-releasing hormone (GHRH), somatostatin (Ss), gonadotropin-releasing hormone $(\mathrm{GnRH})$, corticotrophinreleasing hormone $(\mathrm{CRH})$, and these neurohormones in turn stimulate or inhibit the secretion of hormones of the anterior pituitary (Markakis, 2002; Wells and Murphy, 2003). By contrast, oxytocin and arginine vasopressin (AVP) are produced by magnocellular neurons in the hypothalamus, and unmyelinated axons of these neurons form the hypothalamo-hypophysical tract which ends in the posterior pituitary (Markakis, 2002). Axons of both parvocellular and magnocellular neurons initially project 
through the medial forebrain bundle. Then, axons of parvocellular neurons abruptly exit the bundle, ending in the median eminence, a capillary-rich region in the dorsal infundibulum; whereas axons of magnocellular neurons project further to the posterior pituitary. Given the important roles of the projection of hypothalamic axons to connect with the endocrine system, surprisingly little is known of the mechanisms why axons of hypothalamic neurons end in two separate results. Our studies add understanding to the guidance mechanism of FGF10: at low concentration FGF10 attracts both parvocellular and magnocellular axons; however, at high concentration, parvocellular $\mathrm{TH}+$ axons turn away from FGF10 sources, and magnocellular vasopressin+ axons extend further into FGF10 sources. The concentration dependent functions of FGF10 are not rare in morphogen molecules. For example, Shh (Sonic Hedgehog), a classical morphogen molecule, can act either as an attractive guidance molecule or a repulsive guidance cue on commissural axons in the spinal cord, based on distinct receptor signaling pathways (Kolpak et al., 2005; Parra and Zou, 2010). Thus, we investigate the expression patterns of FGF10 downstream signalling components.

By investigating the expression patterns of FGF10 downstream signalling components, we find evidence for responsive cells in the lateral hypothalamus; importantly, experiments reveal additionally that $F g f r l$ and $F g f r 2$ are also expressed in the mantle region of the lateral hypothalamus, a region contains developing hypothalamic neurosecretory axons. Vascular endothelial cells also express fibroblast growth factor receptors; FGFR1 and FGFR2 pathways are exquisitely sensitive to FGF signals (Oladipupo et al., 2014). Together, these ex vivo observations in chick show that FGF10 exerts concentration dependent functions in angiogenesis and hypothalamic axon guidance, and these effects of FGF10 might mediated by FGFR1/2 signaling pathways.

\section{ACKNOWLEDGEMENT}

This work was supported by National Nature Science Foundation of China (31460259).

\section{Statement of conflict of interest}

There is no conflict of interest.

\section{REFERENCES}

Brewer, J.R., Mazot, P. and Soriano, P., 2016. Genetic insights into the mechanisms of FGF signaling. Genes Dev., 30: 751-771. https://doi.org/10.1101/ gad.277137.115

Eichmann, A. and Thomas, J.L., 2013. Molecular parallels between neural and vascular development. Cold Spring Harb. Perspect. Med., 3: a006551. https://doi.org/10.1101/cshperspect.a006551

Itoh, N. and Ohta, H., 2014. FGF10: a paracrinesignaling molecule in development, disease, and regenerative medicine. Curr. Mol. Med., 14: 504509. https://doi.org/10.2174/156652401466614041 4204829

Kolpak,A.,Zhang, J. andBao,Z.Z., 2005. Sonichedgehog has a dual effect on the growth of retinal ganglion axons depending on its concentration. J. Neurosci. Off. J. Soc. Neurosci., 25: 3432-3441. https://doi. org/10.1523/JNEUROSCI.4938-04.2005

Liu, F., Pogoda, H.M., Pearson, C.A., Ohyama, K., Lohr, H., Hammerschmidt, M. and Placzek, M., 2013. Direct and indirect roles of Fgf3 and Fgf10 in innervation and vascularisation of the vertebrate hypothalamic neurohypophysis. Development, 140: 1111-1122. https://doi.org/10.1242/dev.080226

Liu, F., Li, G., Deng, L., Kuang, B., Li, X., 2017. The roles of FGF10 in vasculogenesis and angiogenesis. Biomed. Res. India, 28: 1329-1332.

Markakis, E.A., 2002. Development of the neuroendocrine hypothalamus. Front. Neuroendocrinol., 23: 257-291. https://doi. org/10.1016/S0091-3022(02)00003-1

Mason, I., 2007. Initiation to end point: the multiple roles of fibroblast growth factors in neural development. Nat. Rev. Neurosci., 8: 583-596. https://doi.org/10.1038/nrn2189

Oladipupo, S.S., Smith, C., Santeford, A., Park, C., Sene, A., Wiley, L.A., Osei-Owusu, P., Hsu, J., Zapata, N., Liu, F., Nakamura, R., Lavine, K.J., Blumer, K.J., Choi, K., Apte, R.S. and Ornitz, D.M, 2014. Endothelial cell FGF signaling is required for injury response but not for vascular homeostasis. Proc. Nat. Acad. Sci. U.S.A., 111: 13379-13384. https://doi.org/10.1073/pnas.1324235111

Parra, L.M. and Zou, Y., 2010. Sonic hedgehog induces response of commissural axons to Semaphorin repulsion during midline crossing. Nat. Neurosci, 13: 29-35. https://doi.org/10.1038/nn.2457

Ribatti, D., 2014. History of research on angiogenesis. Chem. Immunol. Allergy, 99: 1-14. https://doi. org/10.1159/000353311

Sahni, A., Simpson-Haidaris, P.J., Sahni, S.K., Vaday, G.G. and Francis, C.W., 2008. Fibrinogen synthesized by cancer cells augments the proliferative effect of fibroblast growth factor-2 (FGF-2). J. Thromb. Haemost., JTH. 6: 176-183. https://doi.org/10.1111/j.1538-7836.2007.02808.x

Smith, J.C., Hagemann, A., Saka, Y. and Williams, 
P.H., 2008. Understanding how morphogens work. Philos. Trans. R. Soc. Lond. B. Biol. Sci., 363: 1387-1392. https://doi.org/10.1098/rstb.2007.2256 Vesque, C., Ellis, S., Lee, A., Szabo, M., Thomas, P. and Beddington, R., 2000. Development of chick axial mesoderm: specification of prechordal mesoderm by anterior endoderm-derived TGFbeta family signalling. Development, 127: 2795-2809.

Wells, S. and Murphy, D., 2003. Transgenic studies on the regulation of the anterior pituitary gland function by the hypothalamus. Front. Neuroendocrinol., 24: 1126. https://doi.org/10.1016/S0091-3022(02)001036

Yang, X., Liaw, L., Prudovsky, I., Brooks, P.C., Vary, C., Oxburgh, L. and Friesel, R., 2015. Fibroblast growth factor signaling in the vasculature. Curr. Atheroscler. Rep., 17: 509. https://doi.org/10.1007/ s11883-015-0509-6 